\title{
Minimizing Change Aversion for the Google Drive Launch
}

Aaron Sedley

Google, Inc.

1600 Amphitheatre Parkway

Mountain View, CA 94043

United States

asedley@google.com

\section{Hendrik Müller}

Google Australia

5/48 Pirrama Road

Pyrmont, NSW 2009

Australia

hendrikm@google.com
Copyright is held by the author/owner(s)

CHI 2013 Extended Abstracts, April 27-May 2, 2013, Paris, France. ACM 978-1-4503-1952-2/13/04

\begin{abstract}
Change aversion is a natural response, which

technology often exacerbates. Evolutionary changes

can be subtle and occur over many generations. But

Internet users must sometimes deal with sudden,

significant product changes to applications they rely on

and identify with. Despite the best intentions of

designers and product managers, users often

experience anxiety and confusion when faced with a

new interface or changed functionality. While some

change aversion is often inevitable, it can also be

managed and minimized with the right steps. This case

study describes how our understanding of change aversion helped minimize negative effects for the transition of the Google Docs List to Google Drive, a product for file storage in the cloud. We describe actions that allowed for a launch with no aversion.
\end{abstract}

\section{Author Keywords}

Case study; satisfaction; happiness; change aversion; UI design; usability; improvement; user-centered design; user experience; user research; product experience; product launch.

\section{ACM Classification Keywords}

H.5.m. Information interfaces and presentation (e.g. $\mathrm{HCI}$ ): Miscellaneous. 


\section{Introduction}

Change aversion is a state of discomfort and anxiety when something familiar is replaced with something unfamiliar. We are uncomfortable when a product to which we have become accustomed is suddenly changed. We must acclimate to the new version, understand its value, and try to regain the earlier level of comfort. Such changes often cause stress and uncertainty, especially under circumstances we'll explain shortly.

What change aversion is not, however, is getting a product for the first time, such as a teenager's first car or your first smartphone. There is little downside to getting something you have never had before. If the product is very difficult to learn or use, it cannot be because of change aversion, but due to fundamental design flaws, or to expectations exceeding reality.

The case study described in this paper applies our understanding of change aversion to minimize its effects on the transition from the Google Docs List product to Google Drive. Google Drive is a product for storing files in the cloud, editing them in the browser, and accessing them from any device. Google Drive started to replace the Google Docs List in May of 2012.

\section{Change Aversion Basics}

Change aversion is not a revolutionary concept. In fact, the foundations of change aversion are grounded in several well-established psychological principles.

Even before modern psychology, the Greek philosopher Heraclitus wrote that change is ubiquitous, which reminds today's technology companies that standing still is not really an option. If a product does not change over time, its users will and its competitors will.

Early psychologist William James wrote about the power of habit, as if it were a giant flywheel keeping people in their respective social classes. The same effect applies to people's use of products, which generates substantial inertia over time. Any forced changes to well-established habits are prone to cause disruption, and significant effort to regain inertia [1].

The mere exposure effect, identified by Zajonc, showed that familiarity breeds liking. With technology, familiar designs and interactions have a natural advantage over new approaches, at least until a new version is used enough to reap the benefits of familiarity [2].

\section{Kahneman and Tversky, in their famous prospect}

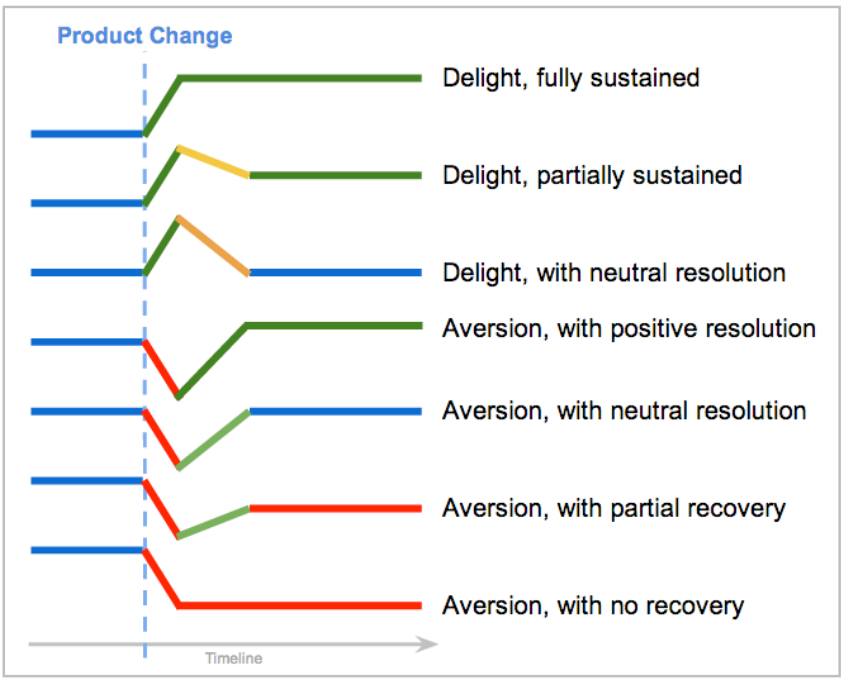

Figure 1: Framework of different reactions to change. 
theory research, found that we place more value on losing what we already have than on gaining the same thing if we don't yet have it. Since changes take away what we have, even if it's replaced by something that is rationally better than what we have lost, it may not be perceived as a net gain - at least not until we feel familiarity and ownership of the new product [3].

Ram and Sheth identified both functional and psychological barriers to innovation. Functional barriers include changes to behavior, an unappealing costbenefit ratio, and uncertainty about performance [4].

In theory, user reactions to change can cover the entire spectrum, from a strongly positive response, to a strongly negative response, and even no response at all. See Figure 1 for a framework of these potential reactions, and their eventual resolution after users acclimate to the changes, the transition period ends and users reach a new baseline state. It is important to consider two dimensions in addition to the positive or negative direction. First is the intensity of the change effect; some changes may generate a slight reaction, others a severe reaction. Secondly, the duration of the change period also varies; some changes are processed quickly by users and a new steady state is rapidly achieved. Other changes have an attenuated effect, due to the time needed to adjust to the new version, and possibly due to lingering public discontent and media attention.

\section{Case Study Description}

We identified key unmet needs and frustrations with the functionality and usability of the Google Docs List by conducting exploratory and evaluative user research. Through several design iterations, combined with usability testing, longitudinal usage analysis, and feedback gathering, we developed a novel product, Google Drive, to replace the Google Docs List.

In parallel, through observation of cases across an array of products and companies, and tracking attitudes toward Google's products, our user experience team conceptualized change aversion as negative reactions toward alterations to a familiar product.

We took several steps to better understand the nature of this phenomenon:

- Considered existing psychological theories

- Identified types of change that influence the degree of aversion

- Explored different patterns of change aversion

- Examined attitudinal data from prior redesign launches

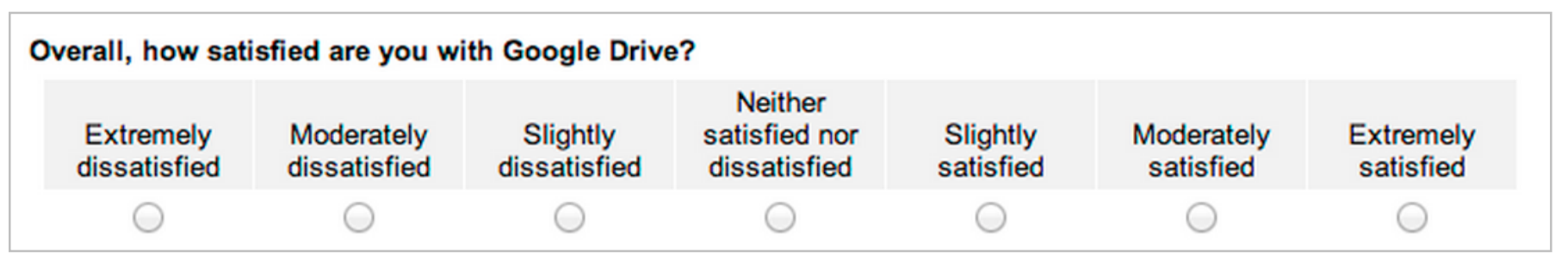

Figure 2. Question used in the Google Drive survey to measure satisfaction trends throughout different launch stages. 
From this inquiry, we developed a framework of actions to minimize change aversion. These actions include:

1. Thoroughly plan the stages of the launch

2. Assess user impact prior to launch

3. Prime users for the upcoming change

4. Explain the benefits of the change

5. Give users transition guidance and support

6. Let users switch between new and old UI

7. Monitor and manage the change over time

8. Let users send feedback directly

9. Address your users' issues quickly

10. Tell your users what you improved

Working with the Google Drive engineering and product management team, we planned to comprehensively apply this framework to the launch of the Google Drive redesign. This was significantly motivated by previous launches that did not apply such change management techniques, resulting in substantial change aversion.

To understand the impact of the redesign on users' attitudes towards the produce (e.g., satisfaction), we implemented a satisfaction tracking survey to continuously measure random samples of users over time. This product survey included a question asking about overall satisfaction, on a 7-point scale from "Extremely dissatisfied" to "Extremely satisfied" (see Figure 2). This contributed an understanding of how efforts to minimize change aversion impacted users' attitudes throughout the redesign launch, and proved the effectiveness of the framework established by the user experience team to minimize change aversion.

\section{Conclusions}

In summary, by understanding the nature of change aversion, we were able to identify and apply several measures to the Google Drive launch to minimize negative effects of the change. Compared to other product redesign launches that did not utilize this change management framework, user perceptions of the Google Drive launch were significantly more positive.

Future research should aim to conduct controlled experiments to isolate the impact of each change management tactic on users' perceptions, and to investigate the effect of survey nonresponse on the representativeness of the data collected prior to and following the launch.

\section{Acknowledgements}

We thank Robin Jeffries and Ed Chi for their comments and suggestions on this case study.

\section{References}

[1] James, W. (1890). Habit. H. Holt.

[2] Zajonc, R. B. (1968). Attitudinal effects of mere exposure. Journal of personality and social psychology, 9(2p2), 1.

[3] Ram, S., \& Sheth, J. N. (1989). Consumer resistance to innovations: the marketing problem and its solutions. Journal of Consumer Marketing, 6(2), 514.

[4] Kahneman, D., \& Tversky, A. (1979). Prospect theory: An analysis of decision under risk.

Econometrica: Journal of the Econometric Society, 263291. 